\title{
Painless thyroiditis in a dupilumab-treated patient
}

\author{
Daramjav Narantsatsral1', Takagi Junko', Iwayama Hideyuki², Inukai Daisuke3, \\ Takama Hiroyuki ${ }^{4}$, Nomura Yuka ${ }^{1}$, Hirase Syo ${ }^{1}$, Morita Hiroyuki' ${ }^{1}$ Otake Kazuo', \\ Ogawa Tetsuya ${ }^{3}$ and Takami Akiyoshi ${ }^{5}$
}

1Division of Endocrinology and Metabolism, Department of Internal Medicine, 2Department of Pediatrics, ${ }^{3}$ Department of Otolaryngology, ${ }^{4}$ Department of Dermatology, and ${ }^{5}$ Department of Hematology, Aichi Medical University School of Medicine, Nagakute, Japan
Correspondence should be addressed to D Narantsatsral Email

daramjav. narantsatsral.026@mail. aichi-med-u.ac.jp

\section{Summary}

Dupilumab an inhibitor of the interleukin (IL)-4R-alpha subunit is used for the treatment of allergic diseases. The patient was a 49-year-old man who received dupilumab for the treatment of severe atopic dermatitis. He presented hyperthyroidism with elevated thyroglobulin and anti-thyroid antibody negativity at 4 months after the initiation of therapy. On scintigraphy, the thyroid radioiodine uptake was low. Ultrasonography showed a diffuse hypoechoic area in the thyroid gland. A pathological study revealed lymphocytic infiltration. The administration of dupilumab was continued because of his atopic dermatitis that showed an excellent response. The patient's hyperthyroidism changed to hypothyroidism 3 weeks later. Six months later his thyroid function normalized without any treatment. We herein describe the case of a patient with atopic dermatitis who developed painless thyroiditis under treatment with dupilumab. To the best of our knowledge, this is the first report of this event in the literature.

\section{Learning points:}

- Dupilumab, a fully human monoclonal antibody that blocks interleukin-4 and interleukin-13, has been shown to be effective in the treatment atopic dermatitis and asthma with eosinophilia.

- Painless thyroiditis is characterized by transient hyperthyroidism and hypothyroidism and recovery without antithyroid treatment.

- This is the first report of painless thyroiditis as an adverse effect of dupilumab, although conjunctivitis and nasopharyngitis are the main adverse effects of dupilumab.

\section{Background}

Autoimmune thyroiditis is characterized with the release of thyroid hormone resulting in transient hyperthyroidism, frequently followed by a hypothyroid phase before recovery of normal thyroid function. Approximately $5-20 \%$ of patients with painless thyroiditis have the characteristic sequence of hyperthyroidism. Painless thyroiditis is associated with specific human leukocyte antigen (HLA) haplotypes, most often HLA-DR3. Medical treatment as interferon alpha, lithium, tyrosine kinase inhibitors, and immune checkpoint inhibitors are possible triggers for painless thyroiditis. Dupilumab is a block of the shared receptor component for interleukin (IL) 4 and IL-13, key drivers of type 2 inflammation in diseases such as atopic dermatitis, asthma, and allergic rhinitis and several adverse effects have been reported in patients treated with dupilumab; however, there are no previous reports on endocrine dysfunction. This is the first report of a patient with painless thyroiditis that appeared in dupilumab treatment. 


\section{Case presentation}

The patient was a 49-year-old man with atopic dermatitis since childhood. The patient had no significant medical history and no family history of thyroid disease including Hashimoto thyroiditis. In May 2019, he was treated with an injection of a $600 \mathrm{mg}$ loading dose of dupilumab followed by the injection of dupilumab (300 mg) every 2 weeks for the treatment of severe atopic dermatitis. At 8 weeks after the initiation of dupilumab, he complained of leg edema, fatigue, and neck discomfort. There was no fever and any symptoms of virus infection. He showed mild hypertension $(140 / 82 \mathrm{mmHg})$, with tachycardia (108 beats/min), and painless goiter.

\section{Investigation}

In the ninth week of dupilumab treatment, laboratory studies revealed the following findings: CRP: $0.06 \mathrm{mg} /$ dL; IgG4: $34.2 \mathrm{mg} / \mathrm{dL}$ (Normal range:11-121); TSH: $0.072 \mathrm{IU} / \mathrm{mL}$; free T3: $4.28 \mathrm{pg} / \mathrm{mL}$; free $\mathrm{T} 4: 1.51 \mathrm{ng} /$ $\mathrm{dL}$; and thyroglobulin: $113.7 \mathrm{ng} / \mathrm{mL}$. A test for antithyroid antibodies was negative (Table 1). Thyroid ultrasonography showed an enlarged thyroid gland, with a diffuse hypoechoic area (size: $2.8 \times 3.4 \times 4.1 \mathrm{~mm}$ ) in the left lobe and smaller lesion with similar characteristics in the right lobe without elevated blood flow, suggesting on-going thyroiditis (Fig. 1A and B). In addition, I ${ }^{131}$ scintigraphy showed a low uptake in the thyroid gland (Fig. 2A and B). The pathological examination of a biopsy specimen of the lesion in left lobe revealed lymphocyte infiltration indicating destructive thyroiditis.

\section{Treatment}

We did not start any anti-thyroid drugs since the clinical examinations indicated painless thyroiditis and his complains gradually disappeared, accompanied by a decrease of T3 and T4. No therapeutic intervention was required for the patient, and the thyroid dysfunction spontaneously improved through the period of hypothyroidism.

\section{Outcome and follow-up}

Dupilumab was continued because the patient's atopic dermatitis showed an excellent response to atopic dermatitis. Follow-up laboratory tests revealed that the function gradually changed to low and transient hypothyroidism (TSH: $8.078 \mathrm{IU} / \mathrm{mL}$; free T3: $2.46 \mathrm{pg} /$ $\mathrm{mL}$; free T4: $0.80 \mathrm{ng} / \mathrm{dL}$ ) 4 months after the initiation of dupilumab. On ultrasonography, a hypoechoic region in the thyroid became invisible 2 months later. His thyroid function normalized without any treatment.

\section{Discussion}

Painless thyroiditis is characterized by transient hyperthyroidism, which is sometimes followed by hypothyroidism, and then recovery, as was observed in our patient. Polymorphism in the Interleukin 4 gene is related to autoimmune thyroiditis (1). In addition, the IL4-positive lymphocyte count is reported to be increased in patients with autoimmune thyroiditis and concurrent non-endocrine disorders (2). IL4 seems to be involved in the pathophysiology of autoimmune thyroiditis. Dupilumab is a human monoclonal IgG4 antibody that inhibits interleukin-4 (IL-4) and interleukin-13 (IL-13) signaling by binding to the IL- $4 \mathrm{R} \alpha$ subunit. The blocking of IL-4R $\alpha$ with dupilumab inhibits IL-4 and IL-13 cytokineinduced inflammatory responses, including the release of proinflammatory cytokines, chemokines, nitric oxide and IgE. The clinical indications of dupilumab include atopic dermatitis, chronic rhinosinusitis with nasal polyposis, and asthma. However, its mechanism of action in asthma has not been definitively established. The adverse effects

Table 1 The laboratory result in the present case.

\begin{tabular}{l}
\hline Test \\
\hline fT3, pg/mL \\
fT4 $n g / d L$ \\
TSH, IU/mL \\
Tg, ng/mL \\
TPOAb, IU/mL \\
TRAb, IU/L \\
CRP, mg/dL \\
IgG4, mg/dL
\end{tabular}

\begin{tabular}{c}
\hline Day 1 \\
\hline 4.28 \\
1.51 \\
0.043 \\
113.7 \\
13.8 \\
0.3 \\
0.06 \\
34.2 \\
\hline
\end{tabular}

\begin{tabular}{l}
\hline Day 32 \\
\hline 2.2 \\
0.62 \\
4.356 \\
34.33 \\
12.0 \\
0.4 \\
\end{tabular}

\begin{tabular}{l}
\hline Day $\mathbf{5 2}$ \\
\hline 2.46 \\
0.8 \\
8.078 \\
42.53 \\
10.3 \\
0.4 \\
\end{tabular}

\begin{tabular}{c} 
Day $\mathbf{8 0}$ \\
\hline 2.65 \\
0.93 \\
4.407 \\
22.2 \\
8.7 \\
0.3
\end{tabular}

\begin{tabular}{c}
\hline Day $\mathbf{1 1 5}$ \\
\hline 2.58 \\
1.01 \\
4.549 \\
18.31 \\
9.7 \\
0.4
\end{tabular}

\begin{tabular}{c}
\hline Day 208 \\
\hline 2.56 \\
0.75 \\
3.297 \\
14.2 \\
11.2 \\
0.5 \\
\end{tabular}

\begin{tabular}{c}
\hline Normal range \\
\hline $2.1-3.1$ \\
$0.75-1.42$ \\
$0.45-3.72$ \\
$<33.7$ \\
$<16$ \\
$<21$ \\
$<30$ \\
$11-121$
\end{tabular}

Blood examination indicated transient hyperthyroidism, followed by hypothyroidism, and recovery. 


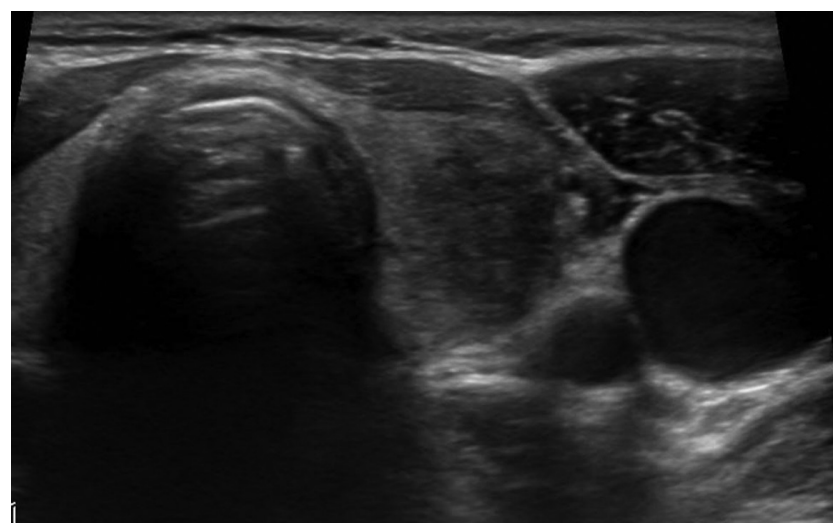

Figure 1

The enlarged thyroid gland, with a diffuse hypoechoic area in the left lobe.

of dupilumab include local injection-site reactions, conjunctivitis, headache, and nasopharyngitis (3). Dry eye syndrome, eosinophilic granulomatosis with polyangiitis, eosinophilic pneumonitis, significant cardiovascular event, and vasculitis are rare $(4,5)$. A meta-analysis of eight randomized controlled trials that was published in January 2018 showed that patients treated with dupilumab had a higher risk of injection-site infection (13.2\%) in comparison to placebo-treated patients (6.5\%) (5), and that among patients with atopic dermatitis, dupilumabtreated patients had a higher incidence of conjunctivitis than placebo-treated patients (5). However previous studies of patients with asthma and chronic sinusitis with nasal polyposis reported that dupilumab-treated patients and placebo-treated patients showed similar incidence of conjunctivitis $(6,7,8)$. In a report on patients with chronic sinusitis with nasal polyposis, dupilumab-treated patients and placebo-treated patients showed a similar incidence of injection-site infection $(8,9)$. Dupilumab moderately increased the risk of injection-site reaction and conjunctivitis in adults with moderate-to-severe atopic dermatitis. This could indicate that dupilumab has another mechanism of action in atopic dermatitis. Painless thyroiditis is diagnosed based on an increased thyroglobulin level, a low radioactive iodine uptake, an increased FT4 level, and a suppressed TSH level. This disorder can occur in patients with autoimmune thyroiditis as a process of the destruction of thyroid tissue. Cytokines, antiviral agents, molecular-targeted therapeutic agents, and immune checkpoint inhibitors including interferon alpha, interleukin-2, tyrosine kinase inhibitors, cytotoxic T-lymphocyte-associated antigen 4 (CTLA-4), are also known to cause painless thyroiditis. However, dupilumab is an IL-4 and IL-13 inhibitor and is not recognized as a

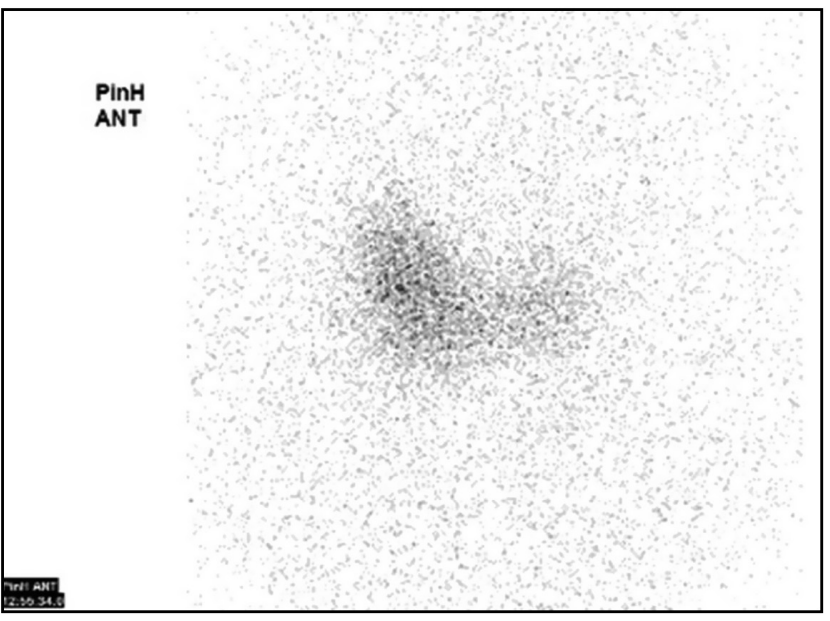

Figure 2

|131-scintigrapy revealed a low uptake in the thyroid gland.

risk factor for thyroiditis or any endocrine disease. Th1 cells and their producing cytokines such as IL-2 play pivotal roles in the development of painless thyroiditis. Dupilumab suppresses Th2 cells through inhibition of IL-4 and IL-13, which leads to the relative dominance of Th1 cells, which may have contributed to the development of painless thyroiditis in the current patient, although this is highly speculative. This may be supported by a previous report (10) in which an increase in the Th1/Th2 ratio, but not in the total number of Th1 cells, was associated with Hashimoto' thyroiditis. In summary, this is the first report of painless thyroiditis related to dupilumab treatment in patients with atopic dermatitis; however, the mechanism underlying this adverse effect is unknown and a single report is not enough to conclude. The investigation is required to clear the mechanisms for the development of silent thyroiditis after dupilumab treatment. Further studies to explore this phenomenon are warranted. The observation of the thyroid function may be useful for patients undergoing treatment with dupilumab.

\section{Declaration of interest}

The authors declare that there is no conflict of interest that could be perceived as prejudicing the impartiality of this case report.

\section{Funding}

This researcher did not receive any specific grant from any funding agency in the public, commercial or not for profit sector.

Patient consen

We obtained written informed consent from the patients. 
Author contribution statement

Both authors contributed equally to the manuscript.

\section{References}

1 Hunt PJ, Marshall SE, Weetman AP, Bell JI, Wass JA \& Welsh KI. Cytokine gene polymorphisms in autoimmune thyroid disease. Journal of Clinical Endocrinology and Metabolism 200085 1984-1988. (https://doi.org/10.1210/jcem.85.5.6588)

2 Santaguida MG, Nardo S, Del Duca SC, Lococo E, Virili C, Gargano L, Lenti L \& Centanni M. Increased interleukin-4-positive lymphocytes in patients with Hashimoto's thyroiditis and concurrent non-endocrine autoimmune disorders. Clinical and Experimental Immunology 2011165 148-154. (https://doi.org/10.1111/j.1365-2249.2011.04419.x)

3 Lee JH, Son SW \& Cho SH. A comprehensive review of the treatment of atopic eczema. Allergy, Asthma and Immunology Research 20168 181-190. (https://doi.org/10.4168/aair.2016.8.3.181)

4 Mitchell K \& Levitt J. Alopecia areata after dupilumab for atopic dermatitis. JAAD Case Reports 20184 143-144. (https://doi. org/10.1016/j.jdcr.2017.11.020)

5 Barnes AC, Blandford AD \& Perry JD. Cicatricial ectropion in a patient treated with dupilumab. American Journal of Ophthalmology Case Reports 20177 120-122. (https://doi.org/10.1016/j. ajoc.2017.06.017)
6 Ou Z, Chen C, Chen A, Yang Y \& Zhou W. Adverse events of dupilumab in adults with moderate-to-severe atopic dermatitis: a meta-analysis. International Immunopharmacology 201854 303-310. (https://doi.org/10.1016/j.intimp.2017.11.031)

7 Bachert C, Mannent L, Naclerio RM, Mullol J, Ferguson BJ, Gevaert P, Hellings P, Jiao L, Wang L, Evans RR, et al. Effect of subcutaneous Dupilumab on nasal polyp burden in patients with chronic sinusitis and nasal polyposis: a randomized clinical trial. JAMA 2016315 469-479. (https://doi.org/10.1001/jama.2015.19330)

8 Wenzel S, Ford L, Pearlman D, Spector S, Sher L, Skobieranda F, Wang L, Kirkesseli S, Rocklin R, Bock B, et al. Dupilumab in persistent asthma with elevated eosinophil levels. New England Journal of Medicine 2013368 2455-2466. (https://doi.org/10.1056/ NEJMoa1304048)

9 Bachert C, Joseph Kh, Desrosiers M, Hellings PW, Amin N, Lee SE, Mullol J, Greos LS, Bosso JV, Laidlaw TM, et al. Efficacy and safety of dupilumab in patients with severe chronic rhinosinusitis with nasal polyps (LIBERTY NP SINUS-24 and LIBERTY NP SINUS-52): results from two multicentre, randomised, double-blind, placebocontrolled, parallel-group phase 3 trials. Lancet 2019394 1638-1650. (https:// doi.org/10.1016/S0140-6736(19)31881-1)

10 Colin IM, Isaac J, Dupret P, Ledant T \& D'Hautcourt JL. Functional lymphocyte subset assessment of the Th1/Th2 profile in patients with autoimmune thyroiditis by flow cytometric analysis of peripheral lymphocytes. Journal of Biological Regulators and Homeostatic Agents $2004 \mathbf{1 8} 72-76$.

Received in final form 8 May 2020

Accepted 26 May 2020 